Von 1000 Soldaten, die sich während des Krieges geschlechtlich ansteckten, waren 740 an Tripper und 260 an Syphilis erkrankt. Das Verhältnis von Tripperkranken zu Syphiliskranken betrug also rund $3: 1$, was eine Zunahme der Syphilis bedeutet, wie sie auch von anderer Seite festgestellt ist. Die Erkrankungen an Ulcus molle waren bei den ausschließlich von der Westfront bzw. von den Garnisonen des Westens stammenden Kranken so außerordentlich gering (etwa sechs bis aoht Fälle), daß ihre Zahl praktisch nicht in Betracht kommt. Von einer Darstellung der Tabellen selbst muß ebenso wie von Berücksichtigung der Literatur aus Raummangel Abstand genommen und lediglich das Ergebnis mitgeteilt werden.

In Berücksichtigung des Berufes der die Ansteckung übertragenden Frauenspersonen ergab sich Folgendes:

\begin{tabular}{|c|c|c|c|}
\hline Berut & $\begin{array}{c}\text { mit Gonorrhoe } \\
\%\end{array}$ & $\underset{\%}{\operatorname{mit} \text { Lues }}$ & $\begin{array}{l}\text { mit einer } \\
\text { Geschlechts- } \\
\text { krankheit } \%\end{array}$ \\
\hline 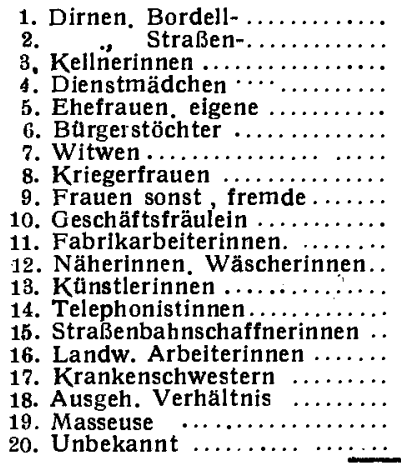 & $\begin{array}{r}5.6 \\
9.6 \\
10.9 \\
10.6 \\
5.3 \\
2.4 \\
1.4 \\
3.2 \\
1.8 \\
7.3 \\
6,1 \\
1.8 \\
0.2 \\
0.1 \\
0.3 \\
0.4 \\
0.1 \\
0.2 \\
\overrightarrow{6.7} \\
\end{array}$ & 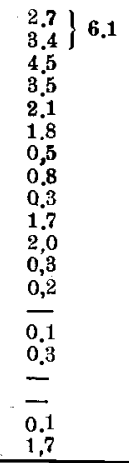 & $\begin{array}{r}8.3 \\
13.0 \\
15.4 \\
14.1 \\
7,4 \\
4.2 \\
1.9 \\
4,0 \\
2.1 \\
9.0 \\
8.1 \\
2.1 \\
0.4 \\
0.1 \\
0.4 \\
0.7 \\
0.1 \\
0.2 \\
0.1 \\
8.4\end{array}$ \\
\hline & 74.0 & $2 f 7$ & 100.0 \\
\hline
\end{tabular}

Von der Gesamtheit der Infektionsträgerinnen in Hinsicht auf ilıren Beruf gibt folgende Zeichnung ein anschauliches Bild.

\title{
Die Ansteckungsquellen der geschlechtskranken Heeresangehörigen während des Krieges.
}

Von Dr. Oscar Gans, Oberarzt d. R., z. Z. Karlsruhe i. B.

Ueber die Infektionsträgerinnen bei den Geschlechtskrankheiten licgen hinsichtlich ihres Berufes bisher nur vereinzelte Zusammenstellungen vor. Aus diesem Grunde habe ich während meiner Lazaretttätigkeit mir die Feststellung der Ansteckungsquellen bei einer Reihe von Soldaten zur Aufgabe gemacht. Um einen einheitlichen Gesichtspunkt zu gewinnen, sind nur diejenigen Ansteckungen berücksichtigt worden, die während des Krieges erfolgten.

Eine derartige Untersuchung hat naturgemäß mit einer Reihe von Fehlerquellen zu rechnen: Nicht zuletzt mit den wissentlich oder aus Gleichgültigkeit wahrheitswidrigen Angaben der Befragten. Ich glaube jedoch für meine Feststellungen eine relative Zu verlässigkeit beanspruchen zu dürfen, da ich bemüht war, durch versohiedene VorsichtsmaBregeln möglichst wahrheitsgemäße Angaben zu erzielen. Ein Hauptpunkt, der vielfach den Befragten zu falschen Aeußerungen veranlaßt, Namensnennung, wurde für beide Teile streng vermieden. Lediglich die Angabe des Berufes der Partnerin wurde verlangt, weiter der Ort der Ansteckung und die Familienverhältnisse des Erkrankten (ledig oder verheiratet). Den Soldaten wurde jedesmal vor der Befragung ein kurzer, aufklärender Vortrag über die Bedeutung der Geschlechtskrankheiten gehalten, daran anschließend über die Möglichkeit der Verhütung gesprochen und sohließlich darauf hingewiesen, daß zur Vorbeugung, wie bei jeder anderen ansteckenden Krankheit, auch hier die Kenntnis der Ansteckungsquellen unbedingt erforderlich sei. Erklärend wurde noch hinzugef ügt, daß auf diese Weise diejenigen Kreise gefunden werden, in welchen die Geschlechtskrankheiten am meisten verbreitet sind. Die Kranken brachten diesem Gedankengange stets Verständnis entgegen. Wenn dann schließlich der Einzelne allein, von mir persönlich im geschlossenen Untersuchungszimmer befragt wurde, hatte man die bestimmte Empfindung, daß der Kranke die Wahrheit sagte, eine Annahme, die auch durch die häufige Angabe ,Unbekannt" gestützt zu werden scheint.

Die Untersuchung erstreckt sich auf 1000 Lazarettinsassen eines Ko. psbezirkes. Neben der Feststellung des Berufes der Partnerin wurde nach dem Orte, an dem die Infektion stattfand, und zwar getrennt für Besatzungsheer (Heimat und Garnison) und Feldheer (auf Urlaub und im Felde bzw. Etappe), gefragt. Eine Trennung nach Feld und Etappe ließ sich mit Sicherheit praktisch nicht durchf ühren, da vielfach die Angaben unsicher waren und die Entscheidung daher auch mehr oder weniger willkürlich hätte sein müssen.

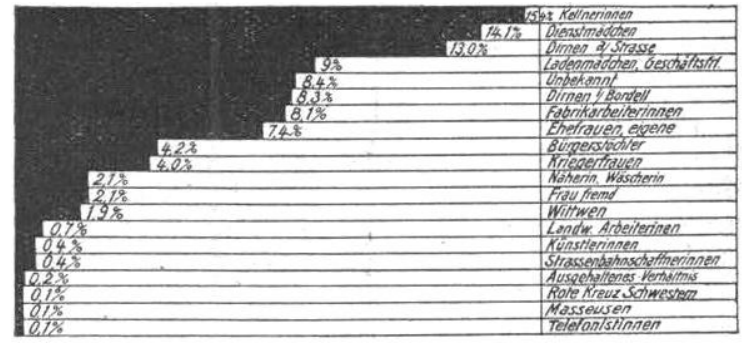
hervor.

Aus dieser Uebersicht heben sich drei große Gruppen augenfällig

1. Kellnerinnen, Dienstmädchen, Straßendirnen.

2. Ladenmä.dchen, Bordelldirnen, Fabrikarbeiterinnen, eigene Ehefrauen.

3. Bürgerstöchter, Kriegerfrauen.

Demnach haben wir die Hauptansteckungsquellen auch während des Krieges bei den Kellnerinnen, Dienstmädchen und Straßendirnen zu suchen.

An zweiter Stelle folgen: Ladenmädchen, Bordelldirnen, Fabrikarbeiterinnen und die eigenen Ehefrauen der Erkrankten.

Die hohe Zahl dieser letzteren Infektionsquelle könnte überraschen, wenn man nickt schon aus den Beobachtungen der Friedenspraxis wüßte, wie häufig die latent verlaufende Gonorrhoe in der Ehe ist, wo sich gewissermaßen die Erreger an die Schleimhäute und die Schleimhaut der Geschlechtsorgane an die Gonokokken gewöhnt hatten. Erst eine längere „Entwöhnung" gibt bei erneutem Verkehr dann Anlaß zum Aufflackern der katarrhalisohen Erscheinungen und damit zum Aufsuchen des Arztes. Immerhin dürfte ein großer Teil dieser Ansteckungen auf extramatrimoniellen Geschlechtsverkehr wohl beider Teile zurückzuführen sein.

Die relativ hohe Beteiligung der Bürgerstörhter und Kriegerfrauen wird den Kenner kaum überraschen; erwähnt sei jedoch, daß die Ansteckung durch Bürgerstöchter in einer großen Zahl der Fälle von Angehörigen des Feldheeres, also im Verkehr in Feindesland, angegeben wurde.

Von der Gesamtzahl der an Gonorrhoe und Lues Frkrankten waren zur Zeit der Ansteckung:

$$
\begin{aligned}
& \text { ledig .....675 }=67,5 \% \\
& \text { verheiratet. } 308=30.8 \% \\
& \text { verwitwet.. 14 }=1.4 \% \\
& \text { geschieden. } \mathbf{3}=0.3 \%
\end{aligned}
$$$$
\text { geschieden } . \quad 3=0.3 \text { ", }
$$

Also unverheiratet 692 , verheiratet 308 . 
Nr. 1

Die Ansteckung erfolgte in 381 Fällen $=38,1 \%$ im Heimatort, in 351 Fällen $=35,1 \%$ in der Garnison, in 128 Fällen $=12,8 \%$ auf Urlaub, und in 140 Fällen im Felde. Mit anderen Worten: Das Besatzungsheer (Heimat und Garnison) ist mit $73,2 \%$, das Feldheer mit $26,8 \%$ an geschlechtlichen Erkrankungen beteiligt, was einem Verhältnis von ungefähr 3:1 entspricht. Von diesen Erkrankungsziffern des Feldheeres entfallen anf die eigentlichen Erkrankungen im Felde, wenn man diese Zahl als verwertbar ansehen darf (s. o.), nur $14 \%$, sodaß in nicht weniger wie $86 \%$ der Fälle die Ansteckung in Heimat, Garnison oder auf Urlaub erfolgt ist.

Diese Feststellung spricht für sich. Sie weist aber auch darauf hin, wo der Hebel anzusetzen ist, $\mathbf{u m}$ mit Aussicht auf Erfolg die Verhütung der Geschlechtskrankheiten durchzuführen. Gewiß ist die zwangsweise Entfernung der geschlechtskrank befundenen Frauen aus den Orten hinter der Front zweckmäßig. Die Hauptrolle der Ansteckung fließt jedoch in der Heimat, in der Garnison, beim Besatzungsheer. Da hier eine zwangsweise Isolierung der kranken Frauen praktisch und urchfihrbar ist, bleibt als einziger Weg die persönliche Prophylaxe des Einzelnen.

Diese Tatsache erneut zu betonen, ist der Zweck der vorliegenden Zeilen. 